\title{
Engineering Protein-Clay Nanosheets Composite Hydrogels with Designed Arginine-Rich Proteins
}

\author{
Shanshan Lv, ${ }^{\dagger, \ddagger}$ Tianyu Duan ${ }^{\dagger}$ and Hongbin $\mathrm{Li}^{*, \dagger}$ \\ †Department of Chemistry \\ The University of British Columbia \\ Vancouver, BC, V6T 1Z1 \\ Canada \\ *State Key Laboratory of Organic-Inorganic Composite Materials \\ Beijing University of Chemical Technology \\ Beijing, 100029 \\ P. R. China
}




\section{Supporting Information}

Table S1. Basic information about the designed proteins.

\begin{tabular}{ccc}
\hline Protein types & Pro17Arg & Pro29Arg \\
\hline Theoretical MW (Da) & 71602 & 86133 \\
Theoretical pI & 4.69 & 4.83 \\
Number of amino acids & 654 & 781 \\
Number of arginine & 17 & 29 \\
Mole ratio of arginine & $1 / 38$ & $1 / 27$ \\
Concentrations $\left(\mathrm{mg} \mathrm{mL}^{-1}\right)$ & 17 & $17 / 33 / 60$ \\
Concentrations of $\mathrm{Gu}^{+}(\mathrm{mM})$ & 4 & $6 / 11 / 20$ \\
\hline
\end{tabular}

Amino acid sequences of Arginine-rich proteins:

Pro17Arg:

MRGSHHHHHHGSMDTYKLILNGKTLKGETTTEAVDAATAEKVFKQYANDNGVDGEWTYDDATKTFT VTERSGGRPSDSYGAPGGGN(RSMDTYKLILNGKTLKGETTTEAVDAATAEKVFKQYANDNGVDGE WTYDDATKTFTVTE) $)_{5}$ RSGGRPSDSYGAPGGGN(RSMDTYKLILNGKTLKGETTTEAVDAATAEKVFK QYANDNGVDGEWTYDDATKTFTVTE) $)_{4}$ RSGGRPSDSYGAPGGGNRSC

\footnotetext{
Pro29Arg:

MRGSHHHHHHGSRRRRRMDTYKLILNGKTLKGETTTEAVDAATAEKVFKQYANDNGVDGEWTYD

DATKTFTVTERSMDTYKLILNGKTLKGETTTEAVDAATAEKVFKQYANDNGVDGEWTYDDATKTFTVT ERSGGRPSDSYGAPGGGN(RSMDTYKLILNGKTLKGETTTEAVDAATAEKVFKQYANDNGVDGEWT YDDATKTFTVTE) ${ }_{5} \mathbf{R S G G R P S D S Y G A P G G G N ( R S M D T Y K L I L N G K T L K G E T T T E A V D A A T A E K V F K Q Y ~}$

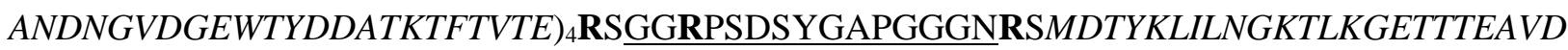
AATAEKVFKQYANDNGVDGEWTYDDATKTFTVTERSRRRRR
}

Note: Sequences in italics correspond to the GB1 sequence, and underlined sequences correspond to the resilin sequence. 